\title{
Polynomial Inequalities in Regions with Piecewise Asymptotically Conformal Curve in the Weighted Lebesgue Space
}

\author{
F.G.ABDULLAYEV ${ }^{*}$, D. ŞIMSSEK ${ }^{2}$, N. SA YPIDINOVA ${ }^{3}$ and Z. TASHPAEVA ${ }^{3}$ \\ ${ }^{1}$ Kyrgyz-Turkish Manas University, Bishkek-KYRGYZSTAN- Mersin University, Mersin-TURKEY \\ ${ }^{2}$ Kyrgyz-Turkish Manas University, Bishkek-KYRGYZSTAN- Selçuk University, Konya-TURKEY \\ ${ }^{3}$ Kyrgyz-Turkish Manas University, Bishkek-KYRGYZSTAN \\ Email: fabdul@mersin.edu.tr; fahreddinabdullayev@gmail.com
}

\begin{abstract}
In this present work, we study the Nikolskii type estimations for algebraic polynomials in the bounded regions with piecewise-asymptotically conformal curve, having interior and exterior zero angles, in the weighted Lebesgue space
\end{abstract}

Keywords: Algebraic polynomials, conformal mapping, assymptotically coformal curve, quasicircle

\section{Introduction}

Let $\mathbb{C}$ be a complex plane; $G \subset \mathbb{C}$ be a bounded region, with $0 \in G$ and Jordan boundary $L:=\partial G$.

Let $\left\{\xi_{j}\right\}_{j=1}^{m}$ be a fixed system of distinct points on curve $L$ located in the positive direction. For some finite region $G^{*} \subset \mathbb{C}$ such that $G \subset G^{*}$ and $z \in G^{*}$, consider a so-called generalized Jacobi weight function $h(z)$ being defined as follows:

$$
h(z):=\prod_{j=1}^{m}\left|z-\xi_{j}\right|^{\gamma_{j}},
$$

where $\gamma_{j}>-1$ for all $j=1,2, \ldots, m$.

For a rectifiable Jordan curve $L$ and for $0<p \leq \infty$, let $\mathcal{L}_{p}(h, L)$ denote the weighted Lebesgue space of complex-valued functions on $L$. Specifically, $f \in \mathcal{L}_{p}(h, L)$ if $f$ is measurable and the following quasinorm (a norm for $1 \leq p \leq \infty$ and a $p$-norm for $0<p<1$ ) is finite:

$$
\begin{aligned}
&\|f\|_{p}:=\|f\|_{\mathcal{L}_{p}(h, L)}:=\left(\int_{L} h(z)|f(z)|^{p}|d z|\right)^{1 / p}, 0<p<\infty ; \\
&\|f\|_{\infty}:=\|f\|_{\mathcal{L}_{\infty}(1, L)}:=e s s \sup _{z \in L}|f(z)|, p=\infty .
\end{aligned}
$$

We denote by $\wp_{n}, n=1,2, \ldots$, the set of all algebraic polynomials $P_{n}(z)$ of degree at most $n \in \mathbb{N}$.

In this work, we study the following Nikolskii-type inequality

$$
\left\|P_{n}\right\|_{\infty} \leq c_{1} \mu_{n}(G, h, p)\left\|P_{n}\right\|_{p}
$$

for some general regions having interior and exterior zero angles of the power type, where $c_{1}=c_{1}(G, p)>0$ is a constant independent of $n, h$ and $P_{n}$, and $\mu_{n}(G, h, p) \rightarrow \infty, n \rightarrow \infty$, depending on the geometrical properties of region $G$ and weight function $h$ in the neighborhood of the points $\left\{\xi_{j}\right\}_{j=1}^{m}$.

The first result of (2)-type, in case $h(z) \equiv 1$ and $L=\{z:|z|=1\}$ for $0<p<\infty$ was found by Jackson [18]. Another classical results similar to (2) belong to Szegö and Zigmund [31]. Suetin [32], [33] investigated this problem with sufficiently smooth Jordan curve. The estimate of (2)-type for $0<p<\infty$ and $h(z) \equiv 1$ when $L$ is a rectifiable Jordan curve was investigated by Mamedhanov [21], [22], Nikolskii [24, pp.122-133], Pritsker [29], Andrievskii [10, Theorem 6] and others. More references regarding the inequality of (2)-type, we can find in Milovanovic et al. [23, Sect.5.3]. 
Further, analogous estimates of (2) for some regions and the weight function $h(z)$ were obtained: in [2] $(p>1)$ and in [25] $\left(p>0, h \equiv h_{0}\right)$ for regions bounded by rectifiable quasiconformal curve having some general properties; in [4] $(p>1)$ for piecewise Dini-smooth curve with interior and exterior cusps; in [3] $(p>1)$ for regions bounded by piecewise smooth curve with exterior cusps but without interior cusps; in [5] $(p>0)$ for regions bounded by piecewise rectifiable quasiconformal curve with cusps; in [6] $(p>0)$ for regions bounded by piecewise quasismooth (by Lavrentiev) curve with cusps.

Now, let's give some definitions and notations.

Let $z_{1}, z_{2}$ be an arbitrary points on $l$ and $l\left(z_{1}, z_{2}\right)$ denotes the subarc of $l$ of shorter diameter with endpoints $z_{1}$ and $z_{2}$. The curve $l$ is a quasicircle if and only if the quantity

$$
\sup _{z_{1}, z_{2} \in l ; z \in l\left(z_{1}, z_{2}\right)} \frac{\left|z_{1}-z\right|+\left|z-z_{2}\right|}{\left|z_{1}-z_{2}\right|}
$$

is bounded [19, p.105]. Following to Lesley [20], we say that the curve $l$ to be said "c-quasiconformal", if the quantity (3) bounded by positive constant $c$, independent from points $z_{1}, z_{2}$ and $z$. At the literature it is possible to find various functional definitions of the quasiconformal curves (see, for example, Def. 3, [26, pp.286-294], [19, p.105], [7, p.81], [27, p.107]).

The Jordan curve $l$ is called asymptotically conformal ([12], [27]), if

$$
\sup _{z_{1}, z_{2} \in l ; z \in l\left(z_{1}, z_{2}\right)} \frac{\left|z_{1}-z\right|+\left|z-z_{2}\right|}{\left|z_{1}-z_{2}\right|} \rightarrow 1, \quad\left|z_{1}-z_{2}\right| \rightarrow 0 .
$$

We will denote this class as $A C$, and will write $G \in A C$, if $L:=\partial G \in A C$.

The asymptotically conformal curves occupy a special place in the problems of the geometric theory of functions of a complex variable. These curves in various problems have been studied by J.M. Anderson, J. Becker and F.D. Lesley [8], E.M.Dyn'kin [13], Ch. Pommerenke, S.E. Warschawski [28], V.Ya. Gutlyanskii, V.I. Ryazanov [14], [15], [16] and others. According to the geometric criteria of quasiconformality of the curves $([7$, p.81], [27, p.107]), every asymptotically conformal curve is a quasicircle. Every smooth curve is asymptotically conformal but corners are not allowed. It is well known that quasicircles can be non-rectifiable (see, for example, [11], [19, p.104]). The same is true for asymptotically conformal curves.

We say that $L \in \widetilde{A C}$, if $L \in A C$ and $L$ is rectifiable. A Jordan arc $\ell$ is called asymptotically conformal arc, when $\ell$ is a part of some asymptotically conformal curve.

Now, we define a new class of regions bounded by piecewise asymptotically conformal curves having interior and exterior zero angles of the power type at the connecting points of boundary arcs.

Throughout this paper, $c, c_{0}, c_{1}, c_{2}, \ldots$ are positive and $\varepsilon_{0}, \varepsilon_{1}, \varepsilon_{2}, \ldots$ are sufficiently small positive constants (generally, different in different relations), which depend on $G$ in general and on parameters inessential for the argument; otherwise, such dependence will be explicitly stated.

For any $k \geq 0$ and $m>k$, notation $i=\overline{k, m}$ means $i=k, k+1, \ldots, m$. For any $i=1,2, \ldots, k=0,1,2$ and $\varepsilon_{1}>0$, we denote by $f_{i}:\left[0, \varepsilon_{1}\right] \rightarrow \mathbb{R}^{+}$and $g_{i}:\left[0, \varepsilon_{1}\right] \rightarrow \mathbb{R}^{+}$twice differentiable functions such that

$$
f_{i}(0)=g_{i}(0)=0, f_{i}^{(k)}(x)>0, g_{i}^{(k)}(x)>0,0<x \leq \varepsilon_{1} .
$$

Definition 1 We say that a Jordan region $G \in A C\left(f_{i}, g_{i}\right)$, for some $f_{i}=f_{i}(x), i=\overline{1, m_{1}}$ and $g_{i}=g_{i}(x), i=\overline{m_{1}+1, m}$, defined as in (5), if $L=\partial G=\bigcup_{i=0}^{m} L_{i}$ is the union of the finite number of asymptotically conformal arcs $L_{i}$, connecting at the points $\left\{z_{i}\right\}_{i=0}^{m} \in L$ and such that $L$ is a locally asymptotically conformal arc at the $z_{0} \in L \backslash\left\{z_{i}\right\}_{i=1}^{m}$ and, in the $(x, y)$ local co-ordinate system with its origin at the $z_{i}, 1 \leq i \leq m$, the following conditions are satisfied:

a) for every $z_{i} \in L, i=\overline{1, m_{1}}, m_{1} \leq m$,

$$
\begin{array}{r}
\left\{z=x+i y:|z| \leq \varepsilon_{1}, c_{11}^{i} f_{i}(x) \leq y \leq c_{12}^{i} f_{i}(x), 0 \leq x \leq \varepsilon_{1}\right\} \subset \bar{G}, \\
\left\{z=x+i y:|z| \leq \varepsilon_{1},|y| \geq \varepsilon_{2} x, 0 \leq x \leq \varepsilon_{1}\right\} \subset \bar{\Omega}
\end{array}
$$

b) for every $z_{i} \in L, i=\overline{m_{1}+1, m}$,

$$
\begin{array}{r}
\left\{z=x+i y:|z|<\varepsilon_{3}, \quad c_{21}^{i} g_{i}(x) \leq y \leq c_{22}^{i} g_{i}(x), 0 \leq x \leq \varepsilon_{3}\right\} \subset \bar{\Omega}, \\
\left\{z=x+i y:|z|<\varepsilon_{3}, \quad|y| \geq \varepsilon_{4} x, 0 \leq x \leq \varepsilon_{3}\right\} \subset \bar{G},
\end{array}
$$


for some constants $-\infty<c_{11}^{i}<c_{12}^{i}<\infty,-\infty<c_{21}^{i}<c_{22}^{i}<\infty$ and $\varepsilon_{s}>0, s=\overline{1,4}$.

Definition 2 We say that a Jordan region $G \in \widetilde{A C}\left(f_{i}, g_{i}\right), f_{i}=f_{i}(x), i=\overline{1, m_{1}}, g_{i}=g_{i}(x), i=$ $\overline{m_{1}+1, m}$, if $G \in A C\left(f_{i}, g_{i}\right)$ and $L:=\partial G$ is rectifiable.

It is clear from Definitions 2 and 1 , that each region $G \in \widetilde{A C}\left(f_{i}, g_{i}\right)$ may have $m_{1}$ interior and $m-m_{1}$ exterior zero angles (with respect to $\bar{G}$ ) at the points $\left\{z_{i}\right\}_{i=1}^{m} \in L$. If a region $G$ does not have interior zero angles $\left(m_{1}=0\right)$ (exterior zero angles $\left.\left(m_{1}=m\right)\right)$, then it is written as $G \in \widetilde{A C}\left(0, g_{i}\right)\left(G \in \widetilde{A C}\left(f_{i}, 0\right)\right)$. If a domain $G$ does not have such angles $(m=0)$, then we will assume that $G$ is bounded by a rectifiable asymptotically conformal curves and in this case we set $\widetilde{A C}(0,0) \equiv \widetilde{A C}$.

Throughout this work, we will assume that the points $\left\{\xi_{i}\right\}_{i=1}^{m} \in L$ defined in $(1)$ and the points $\left\{z_{i}\right\}_{i=1}^{m} \in L$ defined in Definition 2 and 1 coincide. Without loss of generality, we also will assume that the points $\left\{z_{i}\right\}_{i=0}^{m}$ are ordered in the positive direction on the curve $L$ such that $G$ has interior zero angles at the points $\left\{z_{i}\right\}_{i=1}^{m_{1}}$, if $m_{1} \geq 1$ and exterior zero angles at the points $\left\{z_{i}\right\}_{i=m_{1}+1}^{m}$, if $m \geq m_{1}+1$.

\section{Main Results}

Now, we can state our new results. Our first result (Nikolskii-type inequality) is related to the general case. Namely, let region $G$ has $m_{1} \geq 1$ interior zero angles at the points $\left\{z_{i}\right\}_{i=1}^{m_{1}}$ and $m-m_{1}$ exterior zero angles at the points $\left\{z_{i}\right\}_{i=m_{1}+1}^{m}$. In this case, we have the following estimate, i.e. with respect to each points $\left\{z_{i}\right\}_{i=1}^{m}$ :

Theorem 1 Let $p>0 ; G \in \widetilde{A C}\left(f_{i}, g_{i}\right)$, for some $f_{i}(x)=C_{i} x^{1+\alpha_{i}}, \alpha_{i} \geq 0, i=\overline{1, m_{1}}$, and $g_{i}(x)=$ $C_{i} x^{1+\beta_{i}}, \beta_{i}>0, i=\overline{m_{1}+1, m} ; h(z)$ defined as in (1). Then, for any $\gamma_{i}>-1, i=\overline{1, m}$, and $P_{n} \in \wp_{n}, n \in \mathbb{N}$, there exists $c_{1}=c_{1}\left(G, p, \varepsilon, \gamma_{i}, \beta_{i}\right)>0$ such that the following

$$
\left\|P_{n}\right\|_{\infty} \leq c_{1}\left(\sum_{i=1}^{m_{1}} n^{\frac{\widetilde{\left(\gamma_{i}+1\right)(1+\widetilde{\varepsilon})}}{p}}+\sum_{i=m_{1}+1}^{m} n^{\left(\frac{\widetilde{\gamma_{i}}}{1+\beta_{i}}+1\right) \frac{1}{p}+\varepsilon}\right)\left\|P_{n}\right\|_{p}
$$

holds for $\widetilde{\varepsilon}:=\left\{\begin{array}{l}\varepsilon, \text { if } \alpha_{1}=0, \\ 1, \text { if } \alpha_{1} \neq 0,\end{array}\right.$ and arbitrary small $\varepsilon>0$, where $\widetilde{\gamma}_{i}:=\max \left\{0 ; \gamma_{i}\right\}, i=\overline{1, m}$.

Now, for simplicity of our presentations, we assume that: $i=1,2 ; m_{1}=1, m=2$; i.e. our region $G$ has one interior zero (or it does not exist) angle having " $f_{1}$-touching" with $f_{1}(x)=C_{1} x^{1+\alpha_{1}}, \alpha_{1} \geq 0$, at the point $z_{1}$ and exterior zero angle having " $g_{2}$-touching" with $g_{2}(x)=C_{2} x^{1+\beta_{2}}, \beta_{2}>0$, at the point $z_{2}$, for some constants $-\infty<C_{i}<+\infty, C_{i}:=C_{i}\left(c_{i 1}^{i}, c_{i 2}^{i}\right), i=1,2$, where the constants $c_{i j}^{i}, i, j=1,2$, are taken from Definition 2. In this case, combining the terms related to the interior and exterior zero angles, we obtain the following:

Theorem 2 Let $p>0 ; G \in \widetilde{A C}\left(f_{1}, g_{2}\right)$, for some $f_{1}(x)=C_{1} x^{1+\alpha_{1}}, \alpha_{1} \geq 0$, and $g_{2}(x)=C_{2} x^{1+\beta_{2}}, \beta_{2}>$ $0 ; h(z)$ defined as in (1) for $m=2$. Then, for any $\gamma_{i}>-1, i=1,2$, and $P_{n} \in \wp_{n}, n \in \mathbb{N}$, there exists $c_{2}=c_{2}\left(G, p, \varepsilon, \gamma_{i}, \beta_{2}\right)>0$ such that:

$$
\left\|P_{n}\right\|_{\infty} \leq c_{2} A_{n}\left\|P_{n}\right\|_{p}
$$

where

$$
A_{n}:= \begin{cases}n^{\frac{\gamma_{1}+2}{p}}, & \gamma_{1}>\frac{\gamma_{2}}{1+\beta_{2}}-1, \gamma_{2}>1+\beta_{2} ; \\ n^{\left(\frac{\gamma_{2}}{1+\beta_{2}}+1\right) \frac{1}{p}+\varepsilon}, & 0<\gamma_{1} \leq \frac{\gamma_{2}}{1+\beta_{2}}-1, \gamma_{2}>1+\beta_{2} \\ n^{\frac{\gamma_{1}+2}{p}}, & \gamma_{1}>-1,-1<\gamma_{2}<1+\beta_{2} .\end{cases}
$$

In particular, if $\alpha_{1}=0$, i.e. $G$ has only exterior zero angle at the $z_{2}$, then we have: 
Theorem 3 Let $p>0 ; G \in \widetilde{A C}\left(0, g_{2}\right)$, for some $g_{2}(x)=C_{2} x^{1+\beta_{2}}, \beta_{2}>0 ; h(z)$ defined as in (1) for $m=2$. Then, for any $\gamma_{i}>-1, i=1,2$, and $P_{n} \in \wp_{n}, n \in \mathbb{N}$, there exists $c_{3}=c_{3}\left(G, p, \varepsilon, \gamma_{i}, \beta_{2}\right)>0$ such that:

$$
\left\|P_{n}\right\|_{\infty} \leq c_{3} A_{n}\left\|P_{n}\right\|_{p}
$$

where

$$
A_{n}= \begin{cases}n^{\frac{\gamma_{1}+1}{p}+\varepsilon}, & \gamma_{1}>\frac{\gamma_{2}}{1+\beta_{2}}, \gamma_{2}>0 \\ n^{\left(\frac{\gamma_{2}}{1+\beta_{2}}+1\right) \frac{1}{p}+\varepsilon}, & 0<\gamma_{1} \leq \frac{\gamma_{2}}{1+\beta_{2}}, \gamma_{2}>0 \\ n^{\frac{1}{p}+\varepsilon}, & -1<\gamma_{1}, \gamma_{2} \leq 0\end{cases}
$$

The sharpness of the estimations (7)-(9) for some special cases can be discussed by comparing them with the following results:

Remark 1 For the polynomials $P_{n}^{*}(z)=1+z+\ldots+z^{n}$, a) $\left.h^{*}(z) \equiv 1, b\right) h^{* *}(z)=|z-1|^{\gamma}, \gamma>0$, and $L:=\{z:|z|=1\}$, there exists a constant $c_{4}=c_{4}(p)>0$ and $c_{5}=c_{5}\left(h^{* *}, p\right)>0$ such that:

$$
\begin{aligned}
& \text { a) }\left\|P_{n}^{*}\right\|_{\mathcal{L}_{\infty}} \geq c_{4} n^{\frac{1}{p}}\left\|P_{n}^{*}\right\|_{\mathcal{L}_{p}(1, L)}, \quad p>1 ; \\
& \text { b) }\left\|P_{n}^{*}\right\|_{\mathcal{L}_{\infty}} \geq c_{5} n^{\frac{\gamma+1}{p}}\left\|P_{n}^{*}\right\|_{\mathcal{L}_{p}\left(h^{* *}, L\right)}, \quad p>\gamma+1 .
\end{aligned}
$$

\section{Some Aauxiliary Results}

For $a>0$ and $b>0$, we shall use the notations " $a \preceq b$ " (order inequality), if $a \leq c b$ and " $a \asymp b$ " are equivalent to $c_{1} a \leq b \leq c_{2} a$ for some constants $c, c_{1}, c_{2}$ (independent of $a$ and $b$ ) respectively.

Let $G \subset \mathbb{C}$ be a bounded region, and $L:=\partial G$ be a Jordan curve, $\Omega:=\overline{\mathbb{C}} \backslash \bar{G}=\operatorname{ext} L(\overline{\mathbb{C}}:=\mathbb{C} \cup\{\infty\}$. Denote by $w=\Phi(z)$ the univalent conformal mapping of $\Omega$ onto $\Delta:=\{w:|w|>1\}$ with normalization $\Phi(\infty)=\infty, \lim _{z \rightarrow \infty} \frac{\Phi(z)}{z}>0$ and $\Psi:=\Phi^{-1}$.

For $t \geq 1, z \in \mathbb{C}$ and $M \subset \mathbb{C}$, we set:;

$$
\begin{aligned}
L_{t} & :=\{z:|\Phi(z)|=t\} \quad\left(L_{1} \equiv L\right), G_{t}:=\operatorname{int} L_{t}, \Omega_{t}:=\operatorname{ext} L_{t} ; \\
d(z, M) & =\operatorname{dist}(z, M):=\inf \{|z-\zeta|: \zeta \in M\},
\end{aligned}
$$

The following definitions of the $K$-quasiconformal curves are well known (see, for example, [7], [19, p.97] and [30]):

Definition 3 The Jordan arc (or curve) $L$ is called $K$-quasiconformal $(K \geq 1)$, if there is a $K-$ quasiconformal mapping $f$ of the region $D \supset L$ such that $f(L)$ is a line segment (or circle).

Let $F(L)$ denote the set of all sense preserving plane homeomorphisms $f$ of the region $D \supset L$ such that $f(L)$ is a line segment (or circle) and let

$$
K_{L}:=\inf \{K(f): f \in F(L)\},
$$

where $K(f)$ is the maximal dilatation of a such mapping $f . L$ is a quasiconformal curve, if $K_{L}<\infty$, and $L$ is a $K$-quasiconformal curve, if $K_{L} \leq K$.

Lemma 1 [1] Let $L$ be a K-quasiconformal curve, $z_{1} \in L, z_{2}, z_{3} \in \Omega \cap\left\{z:\left|z-z_{1}\right| \preceq d\left(z_{1}, L_{r_{0}}\right)\right\}$; $w_{j}=\Phi\left(z_{j}\right), j=1,2,3$. Then

a) The statements $\left|z_{1}-z_{2}\right| \preceq\left|z_{1}-z_{3}\right|$ and $\left|w_{1}-w_{2}\right| \preceq\left|w_{1}-w_{3}\right|$ are equivalent.

So are $\left|z_{1}-z_{2}\right| \asymp\left|z_{1}-z_{3}\right|$ and $\left|w_{1}-w_{2}\right| \asymp\left|w_{1}-w_{3}\right|$.

b) If $\left|z_{1}-z_{2}\right| \preceq\left|z_{1}-z_{3}\right|$, then

$$
\left|\frac{w_{1}-w_{3}}{w_{1}-w_{2}}\right|^{\varepsilon_{1}} \preceq\left|\frac{z_{1}-z_{3}}{z_{1}-z_{2}}\right| \preceq\left|\frac{w_{1}-w_{3}}{w_{1}-w_{2}}\right|^{c},
$$

where $\varepsilon_{1}<1, c>1,0<r_{0}<1$ are constants, depending on $G$ and $L_{r_{0}}:=\left\{z=\psi(w):|w|=r_{0}\right\}$. 
Lemma 2 [20, p.342] Let $L$ be an asymptotically conformal curve. Then, $\Phi$ and $\Psi$ are Lip $\alpha$ for all $\alpha<1$ in $\bar{\Omega}$ and $\bar{\Delta}$, correspondingly.

Lemma 3 Let $L$ be an asymptotically conformal curve. Then,

$$
\left|\Psi\left(w_{1}\right)-\Psi\left(w_{2}\right)\right| \succeq\left|w_{1}-w_{2}\right|^{1+\varepsilon}
$$

for all $w_{1}, w_{2} \in \bar{\Delta}$ and $\forall \varepsilon>0$.

This fact follows from Lemma 2. We also will use the estimation for the $\Psi^{\prime}$ (see, for example, $[9$, Th.2.8]):

$$
\left|\Psi^{\prime}(\tau)\right| \asymp \frac{d(\Psi(\tau), L)}{|\tau|-1} .
$$

Let $\left\{z_{j}\right\}_{j=1}^{m}$ be a fixed system of the points on $L$ and the weight function $h(z)$ defined as $(1)$.

Lemma 4 Let $L$ be a rectifiable Jordan curve; $h(z)$ defined as in (1). Then, for arbitrary $P_{n}(z) \in \wp_{n}$, any $R>1$ and $n \in \mathbb{N}$, we have

$$
\left\|P_{n}\right\|_{\mathcal{L}_{p}\left(h, L_{R}\right)} \leq R^{n+\frac{1+\tilde{\gamma}}{p}}\left\|P_{n}\right\|_{\mathcal{L}_{p}(h, L)}, p>0
$$

where $\widetilde{\gamma}:=\max \left\{0 ; \gamma_{i}: i=\overline{1, m}\right\}$.

Remark 2 In case of $h(z) \equiv 1$, the estimate (11) has been proved in [17].

\section{Proof of Theorems}

\subsection{Proof of Theorems 1-3.}

Proof. Let $G \in \widetilde{A C}\left(f_{i}, g_{i}\right)$, for some $f_{i}(x)=c_{i} x^{1+\alpha_{i}}, \alpha_{i} \geq 0, i=\overline{1, m_{1}}$, and $g_{i}(x)=c_{i} x^{1+\beta_{i}}, \beta_{i}>0$, $i=\overline{m_{1}+1, m}$, be given. Let $w=\varphi_{R}(z)$ be the univalent conformal mapping of $G_{R}, R>1$, onto the $B$ normalized by $\varphi_{R}(0)=0, \varphi_{R}^{\prime}(0)>0$, and let $\left\{\zeta_{j}\right\}, 1 \leq j \leq m \leq n$, be zeros of $P_{n}(z)$ lying on $G_{R}$. Let

$$
B_{m, R}(z):=\prod_{j=1}^{m} b_{j, R}(z)=\prod_{j=1}^{m} \frac{\varphi_{R}(z)-\varphi_{R}\left(\zeta_{j}\right)}{1-\overline{\varphi_{R}\left(\zeta_{j}\right)} \varphi_{R}(z)}
$$

denote a Blaschke function with respect to zeros $\left\{\zeta_{j}\right\}, 1 \leq j \leq m \leq n$, of $P_{n}(z)$.

Let us set:

$$
Q_{n}(z):=\left[\frac{P_{n}(z)}{B_{m, R}(z)}\right]^{p / 2}, p>0, z \in G_{R}
$$

The function $Q_{n}(z)$ is analytic in $G_{R}$, continuous on $\bar{G}_{R}$ and does not have zeros in $G_{R}$. Then, Cauchy integral representation for the $Q_{n}(z)$ in $G_{R}$ gives:

$$
Q_{n}(z)=\frac{1}{2 \pi i} \int_{L_{R}} Q_{n}(\zeta) \frac{d \zeta}{\zeta-z}, z \in G_{R}
$$

or

$$
\left|\left[\frac{P_{n}(z)}{B_{m, R}(z)}\right]^{p / 2}\right| \leq \frac{1}{2 \pi} \int_{L_{R}}\left|\frac{P_{n}(\zeta)}{B_{m, R}(\zeta)}\right|^{p / 2} \frac{|d \zeta|}{|\zeta-z|} \leq \int_{L_{R}}\left|P_{n}(\zeta)\right|^{p / 2} \frac{|d \zeta|}{|\zeta-z|},
$$

since $\left|B_{m, R}(\zeta)\right|=1$, for $\zeta \in L_{R}$. Let now $z \in L$. Multiplying the numerator and determinator of the integrand by $h^{1 / 2}(\zeta)$, according to the Hölder inequality, we obtain:

$$
\left|\frac{P_{n}(z)}{B_{m, R}(z)}\right|^{p / 2} \leq \frac{1}{2 \pi}\left(\int_{L_{R}} h(\zeta)\left|P_{n}(\zeta)\right|^{p}|d \zeta|\right)^{1 / 2}
$$




$$
\times\left(\int_{L_{R}} \frac{|d \zeta|}{\prod_{j=1}^{m}\left|\zeta-z_{j}\right|^{\gamma_{j}}|\zeta-z|^{2}}\right)^{1 / 2}=: \frac{1}{2 \pi} J_{n, 1} \times J_{n, 2}
$$

where

$$
J_{n, 1}:=\left(\int_{L_{R}} h(\zeta)\left|P_{n}(\zeta)\right|^{p}|d \zeta|\right)^{1 / 2}, J_{n, 2}:=\left(\int_{L_{R}} \frac{|d \zeta|}{\prod_{j=1}^{m}\left|\zeta-z_{j}\right|^{\gamma_{j}}|\zeta-z|^{2}}\right)^{1 / 2} .
$$

Then, since $\left|B_{m, R}(z)\right|<1$, for $z \in L$, from Lemma 4, we have:

$$
\left|P_{n}(z)\right| \preceq\left(J_{n, 1} \cdot J_{n, 2}\right)^{2 / p} \preceq\left\|P_{n}\right\|_{p} \cdot J_{n, 2}^{2 / p}, z \in L .
$$

To estimate the integral $J_{n, 2}$, we introduce:

$$
w_{j}:=\Phi\left(z_{j}\right), \varphi_{j}:=\arg w_{j}, L_{R}^{j}:=L_{R} \cap \bar{\Omega}^{j}, j=\overline{1, m},
$$

where $\Omega^{j}:=\Psi\left(\Delta_{j}^{\prime}\right)$

$$
\begin{aligned}
& \Delta_{1}^{\prime}:=\left\{t=\operatorname{Re}^{i \theta}: R>1, \frac{\varphi_{m}+\varphi_{1}}{2} \leq \theta<\frac{\varphi_{1}+\varphi_{2}}{2}\right\}, \\
& \Delta_{m}^{\prime}:=\left\{t=R e^{i \theta}: R>1, \frac{\varphi_{m-1}+\varphi_{m}}{2} \leq \theta<\frac{\varphi_{m}+\varphi_{1}}{2}\right\} .
\end{aligned}
$$

and, for $j=\overline{2, m-1}$

$$
\Delta_{j}^{\prime}:=\left\{t=R e^{i \theta}: R>1, \frac{\varphi_{j-1}+\varphi_{j}}{2} \leq \theta<\frac{\varphi_{j}+\varphi_{j+1}}{2}\right\} .
$$

Then, we get

$$
J_{n, 2}^{2}=\sum_{i=1}^{m} \int_{L_{R}^{i}} \frac{|d \zeta|}{\prod_{j=1}^{m}\left|\zeta-z_{j}\right|^{\gamma_{j}}|\zeta-z|^{2}} \asymp \sum_{i=1}^{m} \int_{L_{R}^{i}} \frac{|d \zeta|}{\left|\zeta-z_{i}\right|^{\gamma_{i}}|\zeta-z|^{2}}=: \sum_{i=1}^{m} J_{n, 2}^{i},
$$

where

$$
J_{n, 2}^{i}:=\int_{L_{R}^{i}} \frac{|d \zeta|}{\left|\zeta-z_{i}\right|^{\gamma_{i}}|\zeta-z|^{2}}, i=\overline{1, m}
$$

since the points $\left\{z_{j}\right\}_{j=1}^{m} \in L$ are distinct. It remains to estimate the integrals $J_{n, 2}^{i}$ for each $i=\overline{1, m}$. For simplicity of our next calculations, we assume that:

$$
i=1,2 ; m_{1}=1, m=2 ; \quad z_{1}=-1, z_{2}=1 ;(-1,1) \subset G ; R=1+\frac{\varepsilon_{0}}{n},
$$

and let local co-ordinate axis in Definitions 1 and 2 is parallel to $O X$ and $O Y$ in the $O X Y$ coordinate system; $L=L^{+} \cup L^{-}$, where $L^{+}:=\{z \in L: \operatorname{Im} z \geq 0\}, L^{-}:=\{z \in L: \operatorname{Im} z<0\}$. Let $w^{ \pm}:=$ $\left\{w=e^{i \theta}: \theta=\frac{\varphi_{1} \pm \varphi_{2}}{2}\right\}, z^{ \pm} \in \Psi\left(w^{ \pm}\right)$and $L^{i}$ an arcs, connecting the points $z^{+}, z_{i}, z^{-} \in L ; L^{i, \pm}:=$ $L^{i} \cap L^{ \pm}, i=1,2$. Let $z_{0}$ be taken as an arbitrary point on $L^{+}$(or on $L^{-}$subject to the chosen direction). For simplicity, without loss of generality, we assume that $z_{0}=z^{+}\left(z_{0}=z^{-}\right)$.

Analogously to the previous notations, we introduce the following: $L_{R}=L_{R}^{+} \cup L_{R}^{-}$, where $L_{R}^{+}:=$ $\left\{z \in L_{R}: \operatorname{Im} z \geq 0\right\}, L_{R}^{-}:=\left\{z \in L_{R}: \operatorname{Im} z<0\right\} ;$ Let $w_{R}^{ \pm}:=\left\{w=R e^{i \theta}: \theta=\frac{\varphi_{1} \pm \varphi_{2}}{2}\right\}, z_{R}^{ \pm} \in \Psi\left(w_{R}^{ \pm}\right)$.We set: $z_{i, R} \in L_{R}$, such that $d_{i, R}=\left|z_{i}-z_{i, R}\right|$ and $\zeta^{ \pm} \in L^{ \pm}$, such that $d\left(z_{2, R}, L^{2} \cap L^{ \pm}\right):=d\left(z_{2, R}, L^{ \pm}\right) ; z_{i}^{ \pm}$ $:=\left\{\zeta \in L^{i}:\left|\zeta-z_{i}\right|=c_{i} d\left(z_{i}, L_{R}\right)\right\}, z_{i, R}^{ \pm}:=\left\{\zeta \in L_{R}^{i}:\left|\zeta-z_{i, R}\right|=c_{i} d\left(z_{i, R}, L_{R}\right)\right\}, w_{i, R}^{ \pm}=\Phi\left(z_{i, R}^{ \pm}\right)$. Let 
$L_{R}^{i}, i=1,2$, denote arcs, connecting the points $z_{R}^{+}, z_{i, R}, z_{R}^{-} \in L_{R}, L_{R}^{i, \pm}:=L_{R}^{i} \cap L_{R}^{ \pm}$and $l_{i, R}^{ \pm}\left(z_{i, R}^{ \pm}, z_{R}^{ \pm}\right)$ denote arcs, connecting the points $z_{i, R}^{ \pm}$with $z_{R}^{ \pm}$, respectively and $\left|l_{i, R}^{ \pm}\right|:=$mes $l_{i, R}^{ \pm}\left(z_{i, R}^{ \pm}, z_{R}^{ \pm}\right), i=1,2$. We denote:

$$
\begin{aligned}
& S_{1, R}^{i, \pm}:=\left\{\zeta \in L_{R}^{i, \pm}:\left|\zeta-z_{i}\right|<c_{i} d_{i, R}\right\} \\
& S_{2, R}^{i, \pm}:=\left\{\zeta \in L_{R}^{i, \pm}: c_{i} d_{i, R} \leq\left|\zeta-z_{i}\right| \leq\left|l_{i, R}^{ \pm}\right|\right\}, \mathcal{F}_{j, R}^{i, \pm}:=\Phi\left(S_{j, R}^{i, \pm}\right) ; \\
& S_{1}^{i, \pm}:=\left\{\zeta \in L^{i, \pm}:\left|\zeta-z_{i}\right|<c_{i} d_{i, R}\right\} \\
& S_{2}^{i, \pm}:=\left\{\zeta \in L^{i, \pm}: c_{i} d_{i, R} \leq\left|\zeta-z_{i}\right| \leq\left|l_{i, R}^{ \pm}\right|\right\}, \mathcal{F}_{j}^{i, \pm}:=\Phi\left(S_{j}^{i, \pm}\right), i, j=1,2 .
\end{aligned}
$$

Taking into consideration these designations and replacing the variable $\tau=\Phi(\zeta)$, from (10) and (15), we have:

$$
\begin{aligned}
J_{n, 2}^{i} & \asymp \sum_{i, j=1}^{2} \int_{\mathcal{F}_{j, R}^{i,+} \cup \mathcal{F}_{j, R}^{i,-}} \frac{\left|\Psi^{\prime}(\tau)\right||d \tau|}{\left|\Psi(\tau)-\Psi\left(w_{i}\right)\right|^{\gamma_{i}}\left|\Psi(\tau)-\Psi\left(w^{\prime}\right)\right|^{2}} \\
& \asymp \sum_{i, j=1}^{2} \int_{\mathcal{F}_{j, R}^{i,+} \cup \mathcal{F}_{j, R}^{i,-}} \frac{d(\Psi(\tau), L)|d \tau|}{\left|\Psi(\tau)-\Psi\left(w_{i}\right)\right|^{\gamma_{i}}\left|\Psi(\tau)-\Psi\left(w^{\prime}\right)\right|^{2}(|\tau|-1)} \\
& =: \sum_{i, j=1}^{2}\left[J\left(\mathcal{F}_{j, R}^{i,+}\right)+J\left(\mathcal{F}_{j, R}^{i,-}\right)\right] .
\end{aligned}
$$

So, we need to evaluate the integrals $J\left(\mathcal{F}_{j, R}^{i,+}\right)$ and $J\left(\mathcal{F}_{j, R}^{i,-}\right)$ for each $i, j=1,2$. For this, we will continue in the following manner. Let

$$
\left\|P_{n}\right\|_{\infty}=:\left|P_{n}\left(z^{\prime}\right)\right|, z^{\prime} \in L
$$

and let $w^{\prime}=\Phi\left(z^{\prime}\right)$. There are two possible cases: the point $z^{\prime}$ may lie on $L^{1}$ or $L^{2}$.

1) Suppose first that $z^{\prime} \in L^{1}$. If $z^{\prime} \in S_{i}^{1, \pm}$, then $w^{\prime} \in \mathcal{F}_{i}^{1, \pm}$, for $i=1,2$. Consider the individual cases.

1.1) If $z^{\prime} \in S_{1}^{1, \pm}$, then $w^{\prime} \in \mathcal{F}_{1}^{1, \pm}$ and

$$
\begin{gathered}
J\left(\mathcal{F}_{1, R}^{1,+}\right)+J\left(\mathcal{F}_{1, R}^{1,-}\right) \\
\preceq n \int_{\mathcal{F}_{1, R}^{1,+} \cup \mathcal{F}_{1, R}^{1,-}} \frac{|d \tau|}{\left[\min \left\{\left|\Psi(\tau)-\Psi\left(w_{1}\right)\right| ;\left|\Psi(\tau)-\Psi\left(w^{\prime}\right)\right|\right\}\right]^{\gamma_{1}+1}} \\
\preceq n \int_{\mathcal{F}_{1, R}^{1,+} \cup \mathcal{F}_{1, R}^{1,-}} \frac{|d \tau|}{\left[\min \left\{\left|\tau-w_{1}\right| ;\left|\tau-w^{\prime}\right|\right\}\right]^{\left(\gamma_{1}+1\right)(1+\widetilde{\varepsilon})}} \preceq n^{\left(\gamma_{1}+1\right)(1+\widetilde{\varepsilon})},
\end{gathered}
$$

for $\gamma_{1}>0$, and

$$
\begin{gathered}
J\left(\mathcal{F}_{1, R}^{1,+}\right)+J\left(\mathcal{F}_{1, R}^{1,-}\right) \preceq n \int_{\mathcal{F}_{1, R}^{1,+} \cup \mathcal{F}_{1, R}^{1,-}} \frac{\left|\Psi(\tau)-\Psi\left(w_{1}\right)\right|^{\left(-\gamma_{1}\right)}|d \tau|}{\left|\Psi(\tau)-\Psi\left(w^{\prime}\right)\right|} \\
\preceq n \int_{\mathcal{F}_{1, R}^{1,+} \cup \mathcal{F}_{1, R}^{1,-}} \frac{|d \tau|}{\left|\Psi(\tau)-\Psi\left(w^{\prime}\right)\right|} \preceq n \int_{\mathcal{F}_{1, R}^{1,+} \cup \mathcal{F}_{1, R}^{1,-}} \frac{|d \tau|}{\left|\tau-w^{\prime}\right|^{1+\widetilde{\varepsilon}}} \preceq n^{1+\widetilde{\varepsilon}},
\end{gathered}
$$

for $-1<\gamma_{1} \leq 0$;

1.2) If $z^{\prime} \in S_{2}^{1, \pm}$, then

$$
J\left(\mathcal{F}_{1, R}^{1,+}\right)+J\left(\mathcal{F}_{1, R}^{1,-}\right) \preceq n \int_{\mathcal{F}_{1, R}^{1,+} \cup \mathcal{F}_{1, R}^{1,-}} \frac{|d \tau|}{\left|\Psi(\tau)-\Psi\left(w_{1}\right)\right|^{\gamma_{1}}\left|\Psi(\tau)-\Psi\left(w^{\prime}\right)\right|}
$$




$$
\preceq n \int_{\mathcal{F}_{1, R}^{1,+} \cup \mathcal{F}_{1, R}^{1,-}} \frac{|d \tau|}{\left[\min \left\{\left|\tau-w_{1}\right| ;\left|\tau-w^{\prime}\right|\right\}\right]^{\left(\gamma_{1}+1\right)(1+\widetilde{\varepsilon})}} \preceq n^{\left(\gamma_{1}+1\right)(1+\widetilde{\varepsilon})},
$$

for all $\gamma_{1}>0$ and

$$
\begin{aligned}
J\left(\mathcal{F}_{1, R}^{1,+}\right)+J\left(\mathcal{F}_{1, R}^{1,-}\right) & \preceq n \int_{\mathcal{F}_{1, R}^{1,+} \cup \mathcal{F}_{1, R}^{1,-}} \frac{\left|\Psi(\tau)-\Psi\left(w_{1}\right)\right|^{\left(-\gamma_{1}\right)}|d \tau|}{\left|\Psi(\tau)-\Psi\left(w^{\prime}\right)\right|} \\
\preceq n & \int_{\mathcal{F}_{1, R}^{1,+} \cup \mathcal{F}_{1, R}^{1,-}} \frac{|d \tau|}{\left|\tau-w^{\prime}\right|^{1+\widetilde{\varepsilon}}} \preceq n^{1+\widetilde{\varepsilon}},
\end{aligned}
$$

for $-1<\gamma_{1} \leq 0$

1.3) If $z^{\prime} \in S_{1}^{1, \pm}$, then

$$
\begin{aligned}
& J\left(\mathcal{F}_{2, R}^{1,+}\right)+J\left(\mathcal{F}_{2, R}^{1,-}\right) \preceq n \int_{\mathcal{F}_{2, R}^{1,+} \cup \mathcal{F}_{2, R}^{1,-}} \frac{|d \tau|}{\left|\Psi(\tau)-\Psi\left(w_{1}\right)\right|^{\gamma_{1}}\left|\Psi(\tau)-\Psi\left(w^{\prime}\right)\right|} \\
& \preceq n \quad \int_{\mathcal{F}_{2, R}^{1,+} \cup \mathcal{F}_{2, R}^{1,-}} \frac{|d \tau|}{\min \left\{\left|\tau-w_{1}\right| ;\left|\tau-w^{\prime}\right|\right\}^{\left(\gamma_{1}+1\right)(1+\widetilde{\varepsilon})}} \preceq n^{\left(\gamma_{1}+1\right)(1+\widetilde{\varepsilon})},
\end{aligned}
$$

for $\gamma_{1}>0$ and

$$
\begin{aligned}
& J\left(\mathcal{F}_{2, R}^{1,+}\right)+J\left(\mathcal{F}_{2, R}^{1,-}\right) \preceq n \int_{\mathcal{F}_{2, R}^{1,+} \cup \mathcal{F}_{2, R}^{1,-}} \frac{\left|\Psi(\tau)-\Psi\left(w_{1}\right)\right|^{\left(-\gamma_{1}\right)}|d \tau|}{\left|\Psi(\tau)-\Psi\left(w^{\prime}\right)\right|} \\
& \preceq n \int_{\mathcal{F}_{2, R}^{1,+} \cup \mathcal{F}_{2, R}^{1,-}} \frac{|d \tau|}{\left|\tau-w^{\prime}\right|^{1+\widetilde{\varepsilon}}} \preceq n^{1+\widetilde{\varepsilon}},
\end{aligned}
$$

for $-1<\gamma_{1} \leq 0$;

1.4) If $z^{\prime} \in S_{2}^{1, \pm}$, then

$$
\begin{aligned}
& J\left(\mathcal{F}_{2, R}^{1,+}\right)+J\left(\mathcal{F}_{2, R}^{1,-}\right) \preceq n \int_{\mathcal{F}_{2, R}^{1,+} \cup \mathcal{F}_{2, R}^{1,-}} \frac{|d \tau|}{\left|\Psi(\tau)-\Psi\left(w_{1}\right)\right|^{\gamma_{1}}\left|\Psi(\tau)-\Psi\left(w^{\prime}\right)\right|} \\
& \preceq n \quad \int_{\mathcal{F}_{2, R}^{1,+} \cup \mathcal{F}_{2, R}^{1,-}} \frac{|d \tau|}{\left[\min \left\{\left|\tau-w_{1}\right| ;\left|\tau-w^{\prime}\right|\right\}\right]^{\left[\gamma_{1}+1\right](1+\widetilde{\varepsilon})}} \preceq n^{\left(\gamma_{1}+1\right)(1+\widetilde{\varepsilon})},
\end{aligned}
$$

for $\gamma_{1}>0$, and

$$
J\left(\mathcal{F}_{2, R}^{1,+}\right)+J\left(\mathcal{F}_{2, R}^{1,-}\right) \preceq n \int_{\mathcal{F}_{2, R}^{1,+} \cup \mathcal{F}_{2, R}^{1,-}} \frac{|d \tau|}{\left|\Psi(\tau)-\Psi\left(w^{\prime}\right)\right|} \preceq n^{1+\widetilde{\varepsilon}},
$$

for $-1<\gamma_{1} \leq 0$. Combining the relations (19)-(26), we obtain:

$$
\sum_{i=1}^{2}\left[J\left(\mathcal{F}_{i, R}^{1,+}\right)+J\left(\mathcal{F}_{i, R}^{1,-}\right)\right] \preceq n^{\left(\gamma_{1}+1\right)(1+\widetilde{\varepsilon})},
$$

for $\gamma_{1}>0$ and

$$
\sum_{i=1}^{2}\left[J\left(\mathcal{F}_{i, R}^{1,+}\right)+J\left(\mathcal{F}_{i, R}^{1,-}\right)\right] \preceq n^{1+\widetilde{\varepsilon}}
$$


for $-1<\gamma_{1} \leq 0$.

Therefore, in case of $z^{\prime} \in L^{1}$ for each $\gamma_{1}>-1$, from (17), (27) and (28) we get:

$$
J_{n, 2}^{1} \preceq n^{\left(\widetilde{\gamma}_{1}+1\right)(1+\widetilde{\varepsilon})} .
$$

2) Now, suppose that $z^{\prime} \in L^{2}$. If $z^{\prime} \in S_{i}^{2, \pm}$, then $w^{\prime} \in \mathcal{F}_{i}^{2, \pm}$, for $i=1,2$. For the estimate of $J_{n, 2}^{i}$ from (17), again we will consider individual cases.

2.1) If $z^{\prime} \in S_{1}^{2, \pm}$, then

$$
\begin{gathered}
J\left(\mathcal{F}_{1, R}^{2,+}\right)+J\left(\mathcal{F}_{1, R}^{2,-}\right)= \\
\preceq n \int_{\mathcal{F}_{1, R}^{2,+}} \frac{|d \tau|}{\left|\Psi(\tau)-\Psi\left(w_{2}\right)\right|^{\gamma_{2}}\left|\Psi(\tau)-\Psi\left(w^{\prime}\right)\right|} \\
+n \int_{\mathcal{F}_{1, R}^{2,-}} \frac{|d \tau|}{\left|\Psi(\tau)-\Psi\left(w_{2}\right)\right|^{\gamma_{2}}\left|\Psi(\tau)-\Psi\left(w^{\prime}\right)\right|},
\end{gathered}
$$

for all $\gamma_{2}>-1$. The last two integrals are evaluated identically. Therefore, we evaluate one of them, say the first. When $\tau \in \mathcal{F}_{1, R}^{2,+}$, for the $\left|\Psi(\tau)-\Psi\left(w^{\prime}\right)\right|$, we obtain:

$$
\begin{aligned}
\left|\Psi(\tau)-\Psi\left(w^{\prime}\right)\right| & \succeq \max \left\{\left|\Psi(\tau)-\Psi\left(w_{2}\right)\right| ;\left|\Psi(\tau)-z_{2}^{+}\right|\right\} \\
& =\left|\Psi(\tau)-\Psi\left(w_{2}\right)\right| \succeq\left|\Psi(\tau)-z_{2}^{+}\right|^{\frac{1}{1+\beta_{2}}} .
\end{aligned}
$$

Then,

$$
\begin{aligned}
J\left(\mathcal{F}_{1, R}^{2,+}\right) & \preceq n \int_{\mathcal{F}_{1, R}^{2,+}} \frac{|d \tau|}{\left|\Psi(\tau)-z_{2}^{+}\right|^{\frac{\gamma_{2}+1}{1+\beta_{2}}}} \preceq n \int_{\mathcal{F}_{1, R}^{2,+}} \frac{|d \tau|}{\left|\tau-w_{2}^{+}\right|^{\frac{\gamma_{2}+1}{1+\beta_{2}}+\varepsilon}} \\
& \preceq \begin{cases}n^{\frac{\gamma_{2}+1}{1+\beta_{2}}+\varepsilon}, & \frac{\gamma_{2}+1}{1+\beta_{2}}>1-\varepsilon, \\
n \ln n, & \frac{\gamma_{2}+1}{1+\beta_{2}}=1-\varepsilon, \\
n, & \frac{\gamma_{2}+1}{1+\beta_{2}}<1-\varepsilon,\end{cases}
\end{aligned}
$$

if $\gamma_{2}>0$, and

$$
J\left(\mathcal{F}_{1, R}^{2,+}\right) \preceq n \int_{\mathcal{F}_{1, R}^{2,+}} \frac{\left|\Psi(\tau)-\Psi\left(w_{2}\right)\right|^{\left(-\gamma_{2}\right)}|d \tau|}{\left|\Psi(\tau)-z_{2}^{+}\right|^{\frac{1}{1+\beta_{2}}}} \preceq n \int_{\mathcal{F}_{1, R}^{2,+}} \frac{|d \tau|}{\left|\tau-w_{2}^{+}\right|^{\frac{1+\varepsilon}{1+\beta_{2}}}} \preceq n^{\frac{1+\varepsilon}{1+\beta_{2}}}
$$

if $-1<\gamma_{2} \leq 0$, and so, in this case, we get:

$$
J\left(\mathcal{F}_{1, R}^{2,+}\right)+J\left(\mathcal{F}_{1, R}^{2,-}\right) \preceq \begin{cases}n^{\frac{\gamma_{2}+1}{1+\beta_{2}}+\varepsilon}, & \frac{\gamma_{2}+1}{1+\beta_{2}}>1-\varepsilon, \\ n \ln n, & \frac{\gamma_{2}+1}{1+\beta_{2}}=1-\varepsilon, \\ n, & \frac{\gamma_{2}+1}{1+\beta_{2}}<1-\varepsilon,\end{cases}
$$

if $\gamma_{2}>0$, and

$$
J\left(\mathcal{F}_{1, R}^{2,+}\right)+J\left(\mathcal{F}_{1, R}^{2,-}\right) \preceq n^{\frac{1+\varepsilon}{1+\beta_{2}}},
$$

if $-1<\gamma_{2} \leq 0$.

2.2) If $z^{\prime} \in S_{2}^{2, \pm}$, then

$$
J\left(\mathcal{F}_{1, R}^{2,+}\right)+J\left(\mathcal{F}_{1, R}^{2,-}\right) \preceq n \int_{\mathcal{F}_{1, R}^{2,+} \cup \mathcal{F}_{1, R}^{2,-}} \frac{|d \tau|}{\left|\Psi(\tau)-\Psi\left(w_{2}\right)\right|^{\gamma^{2}}\left|\Psi(\tau)-\Psi\left(w^{\prime}\right)\right|},
$$


for all $\gamma_{2}>-1$. When $\tau \in \mathcal{F}_{1, R}^{2,+}$ for the $\left|\Psi(\tau)-\Psi\left(w^{\prime}\right)\right|$, we obtain:

$$
\left|\Psi(\tau)-\Psi\left(w^{\prime}\right)\right| \succeq\left|\Psi(\tau)-z_{2}^{+}\right|
$$

and, analogous to previous case, we get:

$$
J\left(\mathcal{F}_{1, R}^{2,+}\right) \preceq n \int_{\mathcal{F}_{1, R}^{2,+}} \frac{|d \tau|}{\left|\Psi(\tau)-\Psi\left(w_{2}\right)\right|^{\gamma_{2}}\left|\Psi(\tau)-z_{2}^{+}\right|} \preceq n \int_{\mathcal{F}_{1, R}^{2,+}} \frac{|d \tau|}{\left|\tau-w_{2}^{+}\right|^{\frac{\gamma_{2}}{1+\beta_{2}}+1+\varepsilon}} \preceq n^{\frac{\gamma_{2}}{1+\beta_{2}}+1+\varepsilon},
$$

if $\gamma_{2}>0$, and

$$
J\left(\mathcal{F}_{1, R}^{2,+}\right) \preceq n \int_{\mathcal{F}_{1, R}^{2,+}} \frac{\left|\Psi(\tau)-\Psi\left(w_{2}\right)\right|^{\left(-\gamma_{2}\right)}|d \tau|}{\left|\Psi(\tau)-z_{2}^{+}\right|} \preceq n \int_{\mathcal{F}_{1, R}^{2,+}} \frac{|d \tau|}{\left|\Psi(\tau)-z_{2}^{+}\right|} \preceq n^{1+\varepsilon},
$$

if $-1<\gamma_{2} \leq 0$. So, in this case we have:

$$
J\left(\mathcal{F}_{1, R}^{2,+}\right)+J\left(\mathcal{F}_{1, R}^{2,-}\right) \preceq n^{\frac{\gamma_{2}}{1+\beta_{2}}+1+\varepsilon},
$$

if $\gamma_{2}>0$, and

$$
J\left(\mathcal{F}_{1, R}^{2,+}\right)+J\left(\mathcal{F}_{1, R}^{2,-}\right) \preceq n^{1+\varepsilon}
$$

if $-1<\gamma_{2} \leq 0$.

2.3) If $z^{\prime} \in S_{1}^{2, \pm}$, then

$$
\begin{gathered}
J\left(\mathcal{F}_{2, R}^{2,+}\right)+J\left(\mathcal{F}_{2, R}^{2,-}\right) \preceq n \int_{\mathcal{F}_{2, R}^{2,+} \cup \mathcal{F}_{2, R}^{2,-}} \frac{|d \tau|}{\left|\Psi(\tau)-\Psi\left(w_{2}\right)\right|^{\gamma_{2}}\left|\Psi(\tau)-\Psi\left(w^{\prime}\right)\right|} \\
\preceq n \int_{\mathcal{F}_{2, R}^{2,+}} \frac{|d \tau|}{\left|\Psi(\tau)-\Psi\left(w_{2}\right)\right|^{\gamma_{2}}\left|\Psi(\tau)-\Psi\left(w^{\prime}\right)\right|}+n \int_{\mathcal{F}_{2,-}^{2,-}} \frac{|d \tau|}{\left|\Psi(\tau)-\Psi\left(w_{2}\right)\right|^{\gamma_{2}}\left|\Psi(\tau)-\Psi\left(w^{\prime}\right)\right|},
\end{gathered}
$$

for $\gamma_{2}>0$. The last two integrals are evaluated identically. Let's estimate first integral. For $\tau \in \mathcal{F}_{2, R}^{2,+}$ and $z^{\prime} \in S_{1}^{2, \pm}$, we have:

$$
\begin{aligned}
& \left|\Psi(\tau)-\Psi\left(w^{\prime}\right)\right| \succeq\left|\Psi(\tau)-z_{2}^{+}\right| \\
& \left|\Psi(\tau)-\Psi\left(w_{2}\right)\right| \succeq d_{2, R} \succeq\left|z_{2, R}-z_{2}^{+}\right|^{\frac{1}{1+\beta_{2}}} \succeq\left(\frac{1}{n}\right)^{\frac{1+\varepsilon}{1+\beta_{2}}}
\end{aligned}
$$

Then,

$$
J\left(\mathcal{F}_{2, R}^{2,+}\right) \preceq n \int_{\mathcal{F}_{2, R}^{2,+}} \frac{|d \tau|}{\left|\Psi(\tau)-z_{2}^{+}\right|^{\gamma_{2}}\left|\Psi(\tau)-z_{2}^{+}\right|} \preceq n^{\frac{\gamma_{2}}{1+\beta_{2}}+1+\varepsilon} \int_{\mathcal{F}_{2, R}^{2,+}} \frac{|d \tau|}{\left|\tau-w_{2}^{+}\right|^{1+\varepsilon}} \preceq n^{\frac{\gamma_{2}}{1+\beta_{2}}+1+\varepsilon},
$$

and so, for $\gamma_{2}>0$, we obtain:

$$
J\left(\mathcal{F}_{2, R}^{2,+}\right)+J\left(\mathcal{F}_{2, R}^{2,-}\right) \preceq n^{\frac{\gamma_{2}}{1+\beta_{2}}+1+\varepsilon},
$$

For $-1<\gamma_{2} \leq 0$, we get:

$$
\begin{gathered}
J\left(\mathcal{F}_{2, R}^{2,+}\right)+J\left(\mathcal{F}_{2, R}^{2,-}\right)=\int_{\mathcal{F}_{2, R}^{2,+} \cup \mathcal{F}_{2, R}^{2,-}} \frac{\left|\Psi(\tau)-\Psi\left(w_{2}\right)\right|^{\left(-\gamma_{2}\right)}\left|\Psi^{\prime}(\tau)\right||d \tau|}{\left|\Psi(\tau)-\Psi\left(w^{\prime}\right)\right|^{2}} \\
\preceq n \int_{\mathcal{F}_{2, R}^{2,+}} \frac{|d \tau|}{\left|\Psi(\tau)-z_{2}^{+}\right|} \preceq n \int_{\mathcal{F}_{2, R}^{2,+}} \frac{|d \tau|}{\left|\tau-w_{2}^{+}\right|^{1+\varepsilon}} \preceq n^{1+\varepsilon},
\end{gathered}
$$


Then, in this case, we have:

$$
J\left(\mathcal{F}_{2, R}^{2,+}\right)+J\left(\mathcal{F}_{2, R}^{2,-}\right) \preceq n^{\frac{\gamma_{2}}{1+\beta_{2}}+1+\varepsilon} .
$$

2.4) If $z^{\prime} \in S_{2}^{2,+}$, then for $\gamma_{2}>0$

$$
\begin{gathered}
J\left(\mathcal{F}_{2, R}^{2,+}\right) \preceq \frac{n}{d_{2, R}^{\gamma_{2}}} \int_{\mathcal{F}_{2, R}^{2,+}} \frac{|d \tau|}{\left|\Psi(\tau)-\Psi\left(w^{\prime}\right)\right|} \\
\preceq n^{1+\frac{\gamma_{2}}{1+\beta_{2}}(1+\varepsilon)} \int_{\mathcal{F}_{2, R}^{2,+}} \frac{|d \tau|}{\left|\tau-w^{\prime}\right|^{1+\varepsilon}} \preceq n^{\frac{\gamma_{2}}{1+\beta_{2}}+1+\varepsilon},
\end{gathered}
$$

and

$$
\begin{gathered}
J\left(\mathcal{F}_{2, R}^{2,-}\right) \preceq \frac{n}{d_{2, R}^{\gamma_{2}}} \int_{\mathcal{F}_{2, R}^{2,+}} \frac{|d \tau|}{\left|\Psi(\tau)-\Psi\left(w^{\prime}\right)\right|} \\
\preceq n^{1+\frac{\gamma_{2}}{1+\beta_{2}}+\varepsilon} \int_{\mathcal{F}_{2,-}^{2,-}} \frac{|d \tau|}{\left|\Psi(\tau)-\Psi\left(w^{\prime}\right)\right|} \preceq n^{1+\frac{\gamma_{2}}{1+\beta_{2}}+\varepsilon} \int_{\mathcal{F}_{2,-}^{2,-}} \frac{|d \tau|}{\left|\tau-w^{\prime}\right|^{1+\varepsilon}} \\
\preceq n^{\frac{\gamma_{2}}{1+\beta_{2}}+1+\varepsilon} .
\end{gathered}
$$

Case of $z^{\prime} \in S_{2}^{2,-}$ is absolutely identical to the case $z^{\prime} \in S_{2}^{2,+}$. If $-1<\gamma_{2} \leq 0$, then

$$
\begin{aligned}
J\left(\mathcal{F}_{2, R}^{2,+}\right) & =\int_{\mathcal{F}_{2, R}^{2,+}} \frac{\left|\Psi(\tau)-\Psi\left(w_{2}\right)\right|^{\left(-\gamma_{2}\right)}\left|\Psi^{\prime}(\tau)\right||d \tau|}{\left|\Psi(\tau)-\Psi\left(w^{\prime}\right)\right|^{2}} \\
& \preceq n \int_{\mathcal{F}_{2, R}^{2,+}} \frac{|d \tau|}{\left|\Psi(\tau)-\Psi\left(w^{\prime}\right)\right|} \preceq n^{1+\varepsilon},
\end{aligned}
$$

and

$$
\begin{aligned}
J\left(\mathcal{F}_{2, R}^{2,-}\right) & =\int_{\mathcal{F}_{2,-}^{2,-}} \frac{\left|\Psi(\tau)-\Psi\left(w_{2}\right)\right|^{\left(-\gamma_{2}\right)}\left|\Psi^{\prime}(\tau)\right||d \tau|}{\left|\Psi(\tau)-\Psi\left(w^{\prime}\right)\right|^{2}} \\
& \preceq n \int_{\mathcal{F}_{2,-}^{2,-}} \frac{|d \tau|}{\left|\Psi(\tau)-\Psi\left(w^{\prime}\right)\right|} \preceq n^{1+\varepsilon} .
\end{aligned}
$$

Combining the estimations (17), (31)-(39), we obtain:

$$
J_{n, 2}^{2} \preceq n^{1+\varepsilon},
$$

for each $-1<\gamma_{2} \leq 0$ and

$$
J_{n, 2}^{2} \preceq n^{\frac{\gamma_{2}}{\left(1+\beta_{2}\right.}+1+\varepsilon},
$$

for each $\gamma_{2}>0$. Combining (40) and (29), for $m_{1}=1, m_{2}=1$, and any $p>0$, we get:

$$
J_{n, 2}^{1}+J_{n, 2}^{2} \preceq n^{1+\widetilde{\varepsilon}}+n^{1+\varepsilon},
$$

for each $-1<\gamma_{1} \leq 0,-1<\gamma_{2} \leq 0$ and

$$
\begin{gathered}
J_{n, 2}^{1}+J_{n, 2}^{2} \\
\preceq n^{\gamma_{1}+1+\widetilde{\varepsilon}}+n^{\frac{\gamma_{2}}{1+\beta_{2}}+1+\varepsilon},
\end{gathered}
$$


for each $\gamma_{1}>0, \gamma_{2}>0$, where $\widetilde{\varepsilon}:=\left\{\begin{array}{l}\varepsilon, \text { if } \alpha_{1}=0, \\ 1, \text { if } \alpha_{1} \neq 0,\end{array}\right.$ and $p>0$. Then, from (12)-(17), (41) and (42), for all $z \in L$, we obtain:

$$
\begin{array}{r}
\left|P_{n}(z)\right| \preceq\left\|P_{n}\right\|_{p} \cdot\left(n^{\frac{\tilde{\gamma}_{1}+1+\widetilde{\varepsilon}}{p}}+n\left(\frac{\tilde{\gamma}_{2}}{1+\beta_{2}}+1\right) \frac{1}{p}+\varepsilon\right) \\
\preceq\left\|P_{n}\right\|_{p} \cdot \begin{cases}n^{\frac{\gamma_{1}+2}{p}}, & \gamma_{1}>\frac{\gamma_{2}}{1+\beta_{2}}-1, \gamma_{2}>1+\beta_{2} ; \\
n^{\frac{\gamma_{1}+2}{p}}, & \gamma_{1}>0,0<\gamma_{2}<1+\beta_{2} ; \\
n^{\left(\frac{\gamma_{2}}{1+\beta_{2}}+1\right) \frac{1}{p}+\varepsilon}, & 0<\gamma_{1} \leq \frac{\gamma_{2}}{1+\beta_{2}}-1, \gamma_{2}>1+\beta_{2} ; \\
n^{\frac{2}{p}}, & -1<\gamma_{1} \leq 0,-1<\gamma_{2}<1+\beta_{2} ;\end{cases}
\end{array}
$$

if $\alpha_{1} \neq 0$, and

$$
\left|P_{n}(z)\right| \preceq\left\|P_{n}\right\|_{p} \cdot \begin{cases}n^{\frac{\gamma_{1}+1}{p}+\varepsilon}, & \gamma_{1}>\frac{\gamma_{2}}{1+\beta_{2}}, \gamma_{2}>0 ; \\ n^{\left(\frac{\gamma_{2}}{1+\beta_{2}}+1\right) \frac{1}{p}+\varepsilon}, & 0<\gamma_{1} \leq \frac{\gamma_{2}}{1+\beta_{2}}, \gamma_{2}>0 ; \\ n^{\frac{1}{p}+\varepsilon}, & -1<\gamma_{1}, \gamma_{2} \leq 0,\end{cases}
$$

if $\alpha_{1}=0$. Therefore, we completed the proof.

Acknowledgments. This work is supported by KTMU Project No: 2016 FBE 13.

\section{References}

1. F.G. Abdullayev, V.V. Andrievskii, On the orthogonal polynomials in the domains with $K$-quasiconformal boundary. Izv. Akad. Nauk Azerb. SSR., Ser. FTM, no.1, pp. 3-7, 1983.

2. F. G. Abdullayev, N. P. Özkartepe, C. D. Gün, Uniform and pointwise polynomial inequalities in regions without cusps in the weighted Lebesgue space, Bulletin of Tbilisi ICMC vol. 18, no. 1, pp. 146-167, 2014.

3. F. G. Abdullayev, C.D. Gün, N.P. Ozkartepe, Inequalities for algebraic polynomials in regions with exterior cusps, J. Nonlinear Funct. Anal. Article ID, no. 3, pp. 1-32, 2015.

4. F.G. Abdullayev, P. Özkartepe, On the growth of algebraic polynomials in the whole complex plane, $J$. Korean Math. Soc. vol. 52, no. 4, pp. 699-725, 2015.

5. F.G. Abdullayev, P. Özkartepe, Uniform and pointwise polynomial inequalities in regions with cusps in the weighted Lebesgue space, Jaen Journal on Approximation, vol. 7, no. 2, pp. 231-261, 2015.

6. F.G.Abdullayev, P. Özkartepe, Polynomial inequalities in Lavrentiev regions with interior and exterior zero angles in the weighted Lebesgue space, Publications de l'Institut Mathématique (Beograd), vol. 100 (114), pp. 209-227, 2016.

7. L. Ahlfors, Lectures on Quasiconformal Mappings. Princeton, NJ: Van Nostrand, 1966.

8. J. M. Anderson, J. Becker, and F. D. Lesley, Boundary values of asymptotically conformal mappings, J. London Math. Soc., vol. 38, pp. 453-462, 1988.

9. V.V. Andrievskii, V.I. Belyi, V.K. Dzyadyk, Conformal invariants in constructive theory of functions of complex plane. World Federation Publ.Com., Atlanta, 1995.

10. V.V. Andrievskii, Weighted Polynomial Inequalities in the Complex Plane, Journal of Approximation Theory, vol. 164, no. 9, pp. 1165-1183, 2012.

11. P.P. Belinskii, General Properties of Quasiconformal Mappings, Nauka, Sib. otd., Novosibirsk, 1974. [in Russian]

12. J. Becker, C. Pommerenke, Uber die quasikonforme Fortsetzung schlichten Funktionen, Math. Z., 1978, 161, 69-80.

13. E.M. Dyn'kin, Nonanalytic symmetry principle and conformal mappings. - St. Petersburg Math. J., vol. 5, pp. 523-544, 1994

14. V. Gutlyanskii, V. Ryazanov, On asymptotically conformal curves, Complex Variables, vol. 25, pp. 357-366, 1994.

15. V. Gutlyanskii, V. Ryazanov, On the local behaviour of quasi-conformal mappings, Izvestiya: Mathematics, vol. 59, no. 3, pp. 471-498, 1995,.

16. V. Ya. Gutlyanskii, V. I. Ryazanov, On quasi-circles and asymptotically conformal circles, Dokl. Ross. Akad. Nauk, vol. 330, no. 5, pp. 546-548, 1993; (English transl., Russian Acad. Sci. Math., vol. 47, pp. 563-566, 1993). 
17. E. Hille, G. Szegö, J.D. Tamarkin, On some generalization of a theorem of A.Markoff, Duke Math., vol. 3, pp. 729-739, 1937,.

18. D. Jackson, Certain problems on closest approximations. Bull. Amer. Math. Soc., vol. 39, pp. 889-9061933.

19. O. Lehto, K.I. Virtanen, Quasiconformal Mapping in the plane, Springer Verlag, Berlin, 1973.

20. F.D. Lesley, Hölder continuity of conformal mappings at the boundary via the strip method, Indiana Univ. Math. J., vol. 31, pp. 341-354, 1982 .

21. D.I.Mamedhanov, Inequalities of S.M.Nikol'skii type for polynomials in the complex variable on curves, Soviet Math.Dokl., vol. 15, pp. 34-37, 1974.

22. D.I. Mamedhanov, On Nikol'skii-type inequalities with new characteristics, Doklady Mathematics, vol. 82, pp. $882-883,2010$

23. G.V. Milovanovic, D.S. Mitrinovic, Th.M. Rassias, Topics in Polynomials: Extremal Problems, Inequalities, Zeros, World Scientific, Singapore, 1994.

24. S.M. Nikolskii, Approximation of Function of Several Variable and Imbeding Theorems, Springer-Verlag, New-York, 1975.

25. N. P. Özkartepe, F. G. Abdullayev, On the interference of the weight and boundary contour for algebraic polynomials in the weighted Lebesgue spaces I. Ukr. Math. J., vol. 68, no. 10, 2016: (Trans. from Ukr. Mat. Zh. vol. 68, no. 10, 2016, pp. 1365-1379).

26. Ch. Pommerenke, Univalent Functions, Göttingen, Vandenhoeck \& Ruprecht, 1975.

27. Ch. Pommerenke, Boundary Behaviour of Conformal Maps. - Springer-Verlag, Berlin, 1992.

28. Ch. Pommerenke, S.E. Warschawski, On the quantitative boundary behavior of conformal maps, Comment. Math. Helv., vol. 57, pp. 107-129, 1982.

29. I. Pritsker, Comparing Norms of Polynomials in One and Several Variables, J. of Math. Anal. and Appl., vol. 216, pp.685-695, 1997.

30. S. Rickman, Characterisation of quasiconformal arcs, Ann. Acad. Sci. Fenn., Ser. A, Mathematica., 1966, 395, $30 \mathrm{p}$.

31. G. Szegö, A. Zigmund, On certain mean values of polynomials, J.Anal. Math., no. 3, pp. 225-244, 1954.

32. P.K. Suetin, The ordinally comparison of various norms of polynomials in the complex domain, Matematicheskie zapiski Uralskogo Gos. Universiteta, vol. 5 no. 4, 1966. (in Russian).

33. P.K. Suetin, On some estimates of the orthogonal polynomials with singularities weight and contour, Sib. Math. J, vol. VIII, no:3, pp. 1070-1078, 1967. (in Russian). 